\title{
Dietary restriction affects lifespan but not cognitive aging in Drosophila melanogaster
}

Joep M.S. Burger ${ }^{1,2}$, Séverine D. Büchel ${ }^{1,3}$ and Tadeusz J. Kawecki ${ }^{1}$

The content of this preprint is identical with the version published in Aging Cell 6:327-335 (2010)

${ }^{1}$ Department of Ecology and Evolution, University of Lausanne, Switzerland

${ }^{2}$ Present address: Statistics Netherlands, Division of Methodology and Quality, Heerlen, the Netherlands, e-mail: j.burger@cbs.nl

${ }^{3}$ Present address: Institute of Integrative Biology, ETH Zürich, Switzerland, email add in proofs

Correspondence: Tadeusz J. Kawecki, Department of Ecology and Evolution, University of Lausanne, Biophore, CH 1015 Lausanne, Switzerland, email

tadeusz.kawecki@unil.ch,phone+41216924161,fax+41216924165

Running title: Dietary restriction and memory

Keywords: caloric restriction, , learning, memory, functional aging, age-related memory impairment, Drosophila

Word count: total 6962, main text: 4900

\section{Summary}

Dietary restriction extends lifespan in a wide variety of animals, including Drosophila, but its relationship to functional and cognitive aging is unclear. Here we study the effects of dietary yeast content on fly performance in an aversive learning task (association between odor and mechanical shock). Learning performance declined at old age, but 50 day old dietary-restricted flies learned as poorly as equal-aged flies maintained on yeastrich diet, even though the former lived on average 9 days (14\%) longer. Furthermore, at the middle age of 21 days, flies on low yeast diets showed poorer short-term $(5 \mathrm{~min})$ memory than flies on rich diet. In contrast, dietary restriction enhanced 60 min memory of young ( 5 day old) flies. Thus, while dietary restriction had complex effects on learning performance in young to middle-aged flies, it did not attenuate aging-related decline of aversive learning performance. These results are consistent with the hypothesis that, in Drosophila, dietary restriction reduces mortality and thus leads to lifespan extension but does not affect the rate with which somatic damage relevant for cognitive performance accumulates with age. 


\section{Introduction}

Depending on the context, aging is defined as age-dependent intrinsic decline in survivorship and fertility, or as decline in an organism's functions. The former, demographic definition stresses the ultimate consequences of aging for Darwinian fitness and allows quantitative comparisons between very different species (Partridge \& Barton 1996). The latter focuses on the underlying proximate mechanisms. In recent decades the search for a single cause of aging has given way to an increasing recognition that the aging phenotype reflects manifold processes of decay and accumulation of damage (Rose 1991; Austad 2004; Arking 2006). This view is consistent with the evolutionary explanations for aging as a consequence of insufficient investment in somatic maintenance, deleterious side effects of alleles improving survivorship and fertility in youth, and accumulation of mutations with deleterious effects manifested late in life (Medawar 1952; Williams 1957; Kirkwood \& Holliday 1979; Rose 1991; Partridge \& Barton 1993). It is thus of interest which aspects of aging share common proximate mechanisms, and which can be decoupled from each other and from demographic aging.

This paper focuses on the diet-mediated relationship between lifespan and the decline of learning ability and memory with age, which we refer to as cognitive aging. Cognitive aging is a major aspect of human aging and has been documented in rodents (e.g., Flood \& Morley 1998), Notobranchius fish (Valenzano et al. 2006), cockroaches (Brown \& Strausfeld 2009), Drosophila (Tamura et al. 2003; Grotewiel et al. 2005; Mery 2007) and C. elegans (Murakami 2007), but its relationship to demographic aging is not very well understood (Grotewiel et al. 2005). In C. elegans long-lived mutants of several genes involved in insulin/IGF signaling show improved ability to associate temperature with food at both young and old age (Murakami 2007). In contrast, some of the same mutants are impaired, at young age, in the ability to associate $\mathrm{NaCl}$ with absence of food (Vellai et al. 2006). In mice, two long-lived mutants with reduced growth hormone/IGF signaling show a slower age-dependent decline in an aversive learning task (inhibitory avoidance; Forshee 2006). One of those mutants also shows a slower performance decline in a spatial learning task (water maze) while in the other this decline is accelerated compared to wildtype controls (Forshee 2006). In contrast, transgenic overexpression of mitochondrial superoxidase dismutase extends mouse lifespan but has no effect on aversive or spatial learning at either young or old age (Hu et al. 2007). Learning performance of long-lived Drosophila mutants has not been reported, but at least one mutant shows a lower decline in learning ability with age with no effect on lifespan (Yamazaki et al. 2007). In turn, natural genetic variation seems to be characterized by a trade-off between lifespan and learning performance at young age in Drosophila: lines artificially selected for high associative learning ability die younger than unselected controls (Burger et al. 2008). Similarly, long-lived populations show poorer learning at young age (Burger et al. 2008). Thus, some genetic changes appear to delay both demographic and cognitive aging, while other studies indicate that the two may be partially decoupled.

Here we focus on the effects of diet on cognitive aging. Mild nutritional stress (dietary or caloric restriction) consistently extends longevity of organisms as different as yeast, nematodes, flies and rodents (e.g. Masoro 2005; Mair \& Dillin 2008; Piper \& Bartke 2008). This lifespan extension is accompanied by an increased resistance to starvation 
and nutritional stress, and by a strong reduction in reproduction (Tatar et al. 2003; Piper et al. 2005). This response is thought to represent an adaptive shift from the usual reproduction mode to a survival mode, characterized by increased investment in somatic maintenance and/or protection against damage (Tatar et al. 2003; Sinclair 2005; Mair \& Dillin 2008), although it may also involve reduction of damage due to nutrient imbalance (Grandison et al. 2009). Although there are some exceptions (Bond et al. 1989; Means et al. 1993; Bellush et al. 1996; Yanai et al. 2004), multiple studies in rodents report a beneficial effect of dietary restriction on learning performance at old age (e.g., Ingram et al. 1987; Stewart et al. 1989; Pitsikas \& Algeri 1992; Gould et al. 1995; Dubey et al. 1996; Magnusson 2001; Patel \& Finch 2002) or even at young age (Hashimoto \& Watanabe 2005). This lessening of age-related cognitive decline parallels attenuation of other aspects of functional aging: dietary restricted rodents not only live longer, but also remain more youthful and less prone to age-related pathologies (Masoro 2005; Piper \& Bartke 2008). In contrast, to what extent dietary restriction also attenuates functional aging in Drosophila remains unclear (Partridge et al. 2005; Bhandari et al. 2007; Burger et al. 2007), and its effects on learning have not been addressed. Insect brains are simpler and anatomically dissimilar to vertebrate brains, and the range of tasks flies can learn is not overly impressive. However, the molecular mechanisms of learning, acting at the level of individual neurons, seem to be highly conserved across bilateral metazoans and involve at least some orthologous genes (Davis 2005). Recognition of this homology has led to an increasing importance of Drosophila melanogaster as a model system in research on learning as well as aging.

Here we report the effects of dietary yeast content on the performance of young and old Drosophila melanogaster in an aversive learning task, in which the flies were tested for avoidance of an odor previously associated with mechanical shock (Mery \& Kawecki 2005). We manipulated the amount of yeast (the source of amino acids) rather than carbohydrates because it is the essential amino acids that mediate lifetime extension under dietary restriction in flies (Mair et al. 2005; Grandison et al. 2009). We hypothesized that dietary restriction would not only extend lifespan, but also attenuate cognitive aging. Therefore, we expected that long-lived dietary restricted flies would show better learning at middle and old ages than flies kept on a rich diet.

Furthermore, learning is costly in terms of reduced resistance to stressful conditions (Mery \& Kawecki 2003; Mery \& Kawecki 2004; Mery \& Kawecki 2005). Based on the interpretation of the response to dietary restriction as an adaptive shift to a frugal, stressresistant survival mode (Tatar et al. 2003; Sinclair 2005), one would expect such costly activities to be suppressed. Such suppression of learning has e.g. been reported in immunologically challenged (but not sick) honey bees (Mallon et al. 2003). We thus also hypothesized that dietary restriction might have a short-term adverse effect on learning performance at young age. Such a diet-mediated trade-off between longevity and youngage learning performance would parallel the evolutionary trade-off reported in flies (Burger et al. 2008). 


\section{Results}

\section{Longevity and fecundity}

To determine dietary yeast content that leads to dietary restriction (i.e., extends lifespan) without causing malnutrition, we studied the demography of mated female flies on diets containing $1 \%, 2 \%, 5 \%$ and $12 \%$ yeast $w / v$. The $1 \%$ yeast diet resulted in chronic malnutrition. Except for the youngest age (where they still presumably could use some of the reserves accumulated in the larval stage), female flies maintained on this diet hardly laid any eggs (Fig. 1A) and died early (Fig. 1B; median lifespan $48.0 \pm 0.6$ days, average \pm SE over replicate cages). As expected, longevity peaked at an intermediate yeast concentration (Fig. 1B,C), with flies on the $12 \%$ diet dying considerably earlier (median lifespan $64.2 \pm 1.5$ days) than those on the $2 \%$ and $5 \%$ diet (median lifespan $73.0 \pm 1.0$ and $77.2 \pm 1.0$, respectively). However, fecundity at all assayed ages increased steeply and monotonically with dietary yeast content (Fig. 1A). This indicates that the $12 \%$ diet was not toxic, nor led to pathological overfeeding. Inspection of age-specific mortality rates indicates that dietary restriction did not reduce the exponential rate with which mortality increases with age, in agreement with previous results from Drosophila (Partridge et al. 2005). In particular, the logistic-Makeham mortality rate curves for $2 \%$ and $12 \%$ diets were roughly parallel on the log scale (Fig. 1C). If anything, the slope parameter tended to be higher on the $2 \%$ diet than on the $12 \% \operatorname{diet}$ (details not shown).

\section{Learning performance}

Does dietary restriction, in addition to increasing lifespan, also slow down the decline in learning performance with age? To address this question, we used a learning assay in which flies are tested for avoidance of an odor previously associated with aversive mechanical shock. We used once-mated females treated in the same way as in the lifespan assay. We first focused on the effect of diet on short-term memory, assayed 5 min after the end of conditioning. We varied the number of conditioning cycles (1-3) because our previous experience had shown that detection of subtle effects on learning may depend on the intensity of conditioning (e.g., Mery et al. 2007b). Diet had no discernible effects on short-term memory of young (5 day old) flies (Fig. 2A, Table 1). However, at the middle age of 21 days flies maintained on the $1 \%$ and $2 \%$ diets showed poorer performance than those kept on the $5 \%$ or $12 \% \operatorname{diet}(P<0.05$, Tukey's test following ANOVA; Fig. 2B, Table 1). Thus, the effects of diet on short-term memory in 21 day old flies were uncorrelated with its effect on lifespan - performance was reduced in both long-lived flies on the $2 \%$ diet and short-lived flies on $1 \%$ diet. This effect was somewhat more pronounced after one or two than after three conditioning cycles, but the interaction between diet and the number of cycles was not significant (Table 1). By 21 days of age less than $2 \%$ of flies had died and the mortality differences among diet treatments were not yet apparent (Fig.1B,C). Therefore we hypothesized that dietary restriction might still have a positive effect at an old age of 50 days. At this age the mortality differences between diet treatments are pronounced, but still less than $10 \%$ of flies have died in the $2 \%$ or $12 \%$ diet treatments (Fig. 1B), and so the results are unlikely to be biased by survival being selective with respect to learning performance. We tested this hypothesis in a separate experiment; even though the old flies showed a 
substantial decline in short-term memory, there was no difference between the $2 \%$ and $12 \%$ diet treatments (Fig. 2C). Thus, the results for 5 min memory do not support the prediction that dietary restricted flies show a slower decline in learning ability with age.

We verified if this conclusion also holds for 60 min memory; this memory stage (based mostly on middle-term memory, Davis 2005) is thought to be particularly affected by aging (Tamura et al. 2003). Contrary to our expectation, dietary restriction improved the 60 min memory performance of young (5 day old) flies (Fig. 3A), but not of middle-aged (21 day old) or old (50 days) flies (Fig. 3B,C). That dietary restriction may improve 60 min memory in young, but not in older flies was supported by a separate experiment, in which 21-day old flies on the $2 \%$ diet performed less well than those on the $12 \%$ diet; there was no difference at 36 days of age (Supporting Information, supplementary figure S1).

Given that dietary restriction improved learning performance in young but not in aged flies, we wondered if this difference was due to the length of exposure to $2 \%$ versus 12 $\%$ diet, which for the young flies was only 4 days. Therefore we performed a diet switch experiment: some flies were maintained on $12 \%$ diet until 17 days old and switched to 2 $\%$ diet for 4 days, while others were switched in an analogous manner from $2 \%$ to $12 \%$ diet. This diet switch had a profound effect on fecundity: 4 days after the switch from the $2 \%$ to $12 \%$ diet the flies laid as many as eggs as those always maintained on the $12 \%$ diet, whereas the opposite switch caused a 6-fold drop in fecundity (Fig. 4A). Yet, the diet switch in either direction had no detectable effects on 60 min memory score of 21day old flies; if anything, a switch from $12 \%$ to $2 \%$ diet tended to reduce the memory scores compared with flies continuously maintained on $12 \% \operatorname{diet}$ (Fig. 4B). Thus, the positive effect of a short-term (4 days) exposure to dietary restriction on 60 min memory seems to be limited to young flies (Fig. 3A).

\section{Unconditioned response to odors}

Differences in learning performance could be due to differences in unconditioned perception of and response to the odorants used in the learning assays, rather than in the learning ability per se. To address this possibility, we studied how the flies responded to the odors when given a choice in the T-maze between the odorant + solvent versus solvent only, in the absence of any previous experience with the odorant. Both odorants (octanol and methylcyclohexanol) are known to be aversive to flies, and young and middle-aged flies strongly avoided both odors, with no difference among diet treatments (Fig. 5 A,B). In contrast, not only was odor avoidance much weaker in 50-day old flies, but flies on $12 \%$ diet showed poorer avoidance of both odors than flies on $2 \% \operatorname{diet}$ (Fig. 5C). However, in an assay of unconditioned preference, when faced with choice between the odorants, 50 -day old flies on the $2 \%$ and $12 \%$ diets showed similar slight preference for methylcyclohexanol (respectively $58 \pm 4 \%$ versus $61 \pm 3 \%$ of flies choosing methylcyclohexanol, $\left.F_{1,7}=0.3, P=0.61\right)$. Thus, despite the weaker absolute aversion of flies from the $12 \%$ diet to both odors, their relative unconditioned odor preference was apparently unaffected by diet. Thus, the differences in learning performance are unlikely to be due to differences in unconditioned odor preference. 


\section{Discussion}

\section{Dietary restriction may improve 60 min memory at young age}

We hypothesized that dietary restriction-induced shift to the "survival mode" may be associated with suppression of costly cognitive processes, leading to poorer learning performance. Contrary to this expectation, we found that young dietary restricted flies showed better learning performance when assayed 60 min after conditioning, but not when assayed $5 \mathrm{~min}$ after conditioning. Differential effects of treatments on different memory phases are not unusual (Tamura et al. 2003; Isabel et al. 2004; Mery et al. 2007a); genetic dissections have identified four distinct forms of memory in fruit flies (Davis 2005). Here, the 5 min learning response is based on short-term memory, while the 60 min response relies mostly on middle-term memory (Davis 2005). Non surprisingly, starvation has been shown to improve performance of flies in learning tasks involving a food reward (Colomb et al. 2009), but the apparent enhancement by dietary restriction of middle-term memory in an aversive task is puzzling. In some rodent studies dietary restriction enhanced the learning performance of young animals (Lukoyanov et al. 2002; Hashimoto \& Watanabe 2005), but most rodent studies (reviewed in the introduction) only found positive effects in older animals. Flies subject to mild nutritional stress might possibly become sensitized to pain caused by the shock, or be generally more alert, but if so, one would expect to observe an effect on short-term memory as well. Alternatively, one can speculate that nutritional stress upregulates their learning behavior as it may help them find food, and this effect generalized to aversive learning. Finally, flies in the dietary restriction-induced "survival mode" may become more riskaverse, remembering dangerous situations longer. However, to explain the results, these mechanisms would have to be restricted to young flies.

Whatever its mechanism, the dietary restriction-induced enhancement of 60 min memory effect is apparently limited to young flies; it does not occur in 21 day old flies by either short-term (4 days) or long-term (20 days) exposure to restricted diet. If anything, 21 day old flies maintained from eclosion on yeast-poor diet showed poorer short-term memory, and in one of two experiments also showed poorer 60 min memory. At least for the shortterm memory this reduction in performance bears no simple relationship to lifespan extension, and rather seems directly related to the nutritional status. Long-lived flies on the $5 \%$ diet and short-lived flies on the $12 \%$ diet both learned well, while long-lived flies kept on the $2 \%$ diet showed the same performance reduction as the malnourished, short-lived flies on the $1 \%$ diet.

Thus, the effects of diet on learning performance in young to middle-aged flies seem complex, depending on age and memory assay. Similar age-dependent effects of diet have been reported for other traits, such as immunity, starvation resistance and coldshock response (Burger et al. 2007). However, in contrast to most traits reported by Burger et al (2007), the effect of dietary restriction on learning performance vanished in old flies. 


\section{Dietary restriction does not attenuate cognitive aging}

Even though learning performance declined at old age, long-lived dietary-restricted flies learned as poorly at old age ( 36 and 50 days), as equal-aged flies maintained on a rich diet. This is not due to lack of sensitivity of the learning assay (e.g., due to saturation of the response); the assay clearly detected the impact of the amount of conditioning (number of cycles). Thus, contrary to our prediction, lifespan extension in dietaryrestricted flies was not associated with delayed cognitive aging. It is possible that a small effect existed but escaped detection (the $95 \%$ combined confidence limits for the difference between $2 \%$ and $12 \%$ diet on the memory score at 50 days of age are -0.051 , 0.086). The predicted effect might also possibly occur in other learning assays, under different dietary restriction regimes, or in different fly strains. Nonetheless, a robust positive effect of dietary restriction on learning performance in aged flies is apparently absent.

A failure of dietary restriction to attenuate aging-related decline has been reported for some other aspects of performance in Drosophila, including negative geotaxis (Bhandari et al. 2007) and resistance to cold stress (Burger et al. 2007). Nonetheless, in our study dietary restriction did attenuate somewhat the decline in odor avoidance in old flies, confirming a non-significant trend reported by Bhandari et al (2007). Dietary restriction has also been reported to improve immunity of old flies to systemic bacterial infections (Burger et al. 2007). In contrast, the loss of resistance to starvation and oxidative stress in old flies is aggravated by dietary restriction (Burger et al. 2007). Thus, while dietary restriction apparently improves some aspects of performance in old flies, those studies and the results presented in this paper strongly suggest that dietary restriction does not generally attenuate cognitive and functional aging in Drosophila. It is possible that dietary restriction alleviates age-related accumulation of somatic damage in a tissue- or function-specific way. However, our results are also consistent with the controversial hypothesis that dietary restriction in Drosophila does not affect the rate at which somatic damage accumulates with age, but rather reduces the sensitivity of survival (and some other functions) to already accumulated damage (Partridge et al. 2005). Demographic support for this hypothesis comes from the fact that low-yeast diet extends lifespan through an acute (short-term) reduction in mortality, rather than by reducing the rate with which mortality increases with age (Mair et al. 2003; Partridge et al. 2005). In agreement with those results, in our study dietary restriction reduced mortality rates at most age classes, but did not reduce the slope of the log mortality rate on age (often used as a measure of demographic aging; e.g., Partridge et al. 2005, although see Rozing \& Westendorp 2008). Molecular evidence indeed indicates that dietary restriction does not affect production of reactive oxygen species (Miwa et al. 2003) or accumulation of somatic DNA damage (Edman et al. 2009).

How to reconcile our results with the overwhelming evidence that dietary restriction attenuates cognitive and functional aging in rodents (reviewed in the introduction)? Because dietary restriction consistently extends lifespan in such a great variety of organisms, it is usually assumed that the mechanisms underlying this phenomenon have been evolutionary conserved. However, if the response to dietary restriction is an adaptation to survive periods of famine, it may have evolved independently in different major taxa as an example of convergent evolution. Even if this is not the case, the details 
of this response have likely been molded by evolution to match the ecology and physiology of the taxon. Hence, even though the demographic consequences of dietary restriction are similar among taxa, it would not necessarily be surprising if the associated functional phenotypes and underlying mechanisms turned out to be different. Better understanding of the selection acting on the response in nature would help to interpret such differences.

\section{Experimental procedures}

\section{Flies and culture conditions}

We used wild-type Drosophila melanogaster flies from an outbred population established from 189 fertile females caught in early October 2006 in a vineyard near Sierre, Switzerland. The population had been maintained at over 1200 individuals in discrete (non-overlapping) generations, in multiple vials with about 250 eggs per $30 \mathrm{ml}$ yeastcornmeal-sucrose medium with $2 \%(\mathrm{w} / \mathrm{v})$ yeast.

We manipulated adult diet by varying the yeast concentration; this is sufficient to manipulate longevity in flies (Chippindale et al. 1993; Mair et al. 2005). We used four diet recipes, with 10, 20, 50 or $120 \mathrm{~g}$ of dry inactive brewer's yeast (Actilife Fitovit, Migros, Switzerland) per liter of water; we refer to these diets as $1 \%, 2 \%, 5 \%$ and $12 \%$ diets, respectively. All diets contained $110 \mathrm{~g}$ of sucrose, $52 \mathrm{~g}$ of cornmeal, $7.9 \mathrm{~g}$ of agar, $2.3 \mathrm{~g}$ of methyl 4-hydroxybenzoate and $8.9 \mathrm{~g}$ of ethanol per liter of water (modified from Min \& Tatar 2006).

All flies used in the experiment were raised as larvae on the $2 \%$ yeast medium at $25^{\circ} \mathrm{C}$. Adults that emerged on days 10-12 from egg were collected for the experiments. Within 24 hours after eclosion, the adults (sexes mixed) were transferred to one of the four diets (see above); unless stated otherwise they were maintained on this diet until the end the experiment. Following the transfer, the flies were given two days to mate, after which (on day 3 from eclosion) they were sexed using $\mathrm{CO}_{2}$ anesthesia. Males were discarded; females were used to assay longevity, fecundity, learning performance and odor avoidance as described below.

We used a longitudinal design: flies of the same cohort were tested at different ages and thus necessarily on different days. Fly behavior tends to vary from day to day e.g. in response to changing atmospheric pressures. To minimize this confounding day effect, all assays were performed on six blocks (cohorts) set up on different days within a 10-day period. Flies for all experiments were maintained at $25^{\circ} \mathrm{C}, 65 \%$ relative humidity and under a 14/10-hour light/dark cycle.

\section{Longevity}

Following mating and sexing, 3-day old females were transferred in groups of 200 to 11 demography cages with a $40 \mathrm{ml}$ vial containing $10 \mathrm{ml}$ food of the appropriate diet. We changed the food and scored the number of dead flies three times per week. Flies that escaped or were stuck in the food but were still alive were treated as censored observations. Cages were rotated throughout the incubator. We measured longevity on 
the four diets, with one cage per block and diet $(24$ cages $=4800$ flies in total). Productmoment survival curves were estimated taking into account censored data; the survival curves were compared with the proportional hazard model (Cox regression) using JMP statistical software (v. 7.0). We used WinModest v. 1.0.2 (Pletcher 1999) to fit mortality models; we report the logistic-Makeham model (Pletcher 1999), which provided the best fit. Estimates of age-specific mortality rates (used for illustration only) were calculated for weekly intervals as $\ln (1-$ number of flies dead within the interval/number of flies alive at the beginning of the interval)/interval length.

\section{Fecundity}

Two days before the fecundity assay each female was transferred singly (using $\mathrm{CO}_{2}$ anesthesia) to $60-\mathrm{ml}$ vial with two young males and $10 \mathrm{ml}$ of the appropriate diet. For females to be assayed at the age of 5 days, this was done immediately following mating and sexing on day 3 from eclosion (see above). Females assayed at older ages were maintained until two days before the assay in the demography cages under the same conditions as used in the longevity assay (i.e., without males). On the day of fecundity assay, each female was transferred together with the males to a new vial. After 24 hours, we discarded the adults and counted the number of eggs laid. Fecundity was measured for five females per block, diet and age; different females were used to assay fecundity at different ages. The effect of these factors on the numbers of eggs laid by individual females was assayed with generalized linear model (as implemented in JMP v. 7.0) assuming the Poisson error distribution and a logarithmic link function.

\section{Learning performance}

We used an olfactory aversive learning assay in which flies were first conditioned to associate an odor with aversive mechanical shock and then tested for the choice between this and another odor in a T-maze (modified from Mery \& Kawecki 2005; Mery et al. 2007b). As in the fecundity assay, flies tested at ages older than 5 days were maintained until two days before the assay in the demography cages.

The conditioning and test procedures were carried out on groups of 50 females; these groups were formed (using $\mathrm{CO}_{2}$ ) two days before the assay but continued to be maintained on their respective diet. On the day of the assay each group of 50 flies was transferred without anesthesia to a 10-ml test tube without food and exposed to one, two or three conditioning cycles. Each conditioning cycle consisted of a $30 \mathrm{~s}$ exposure to one odor accompanied by six bouts of mechanical shock at $5 \mathrm{~s}$ intervals (VWR vortex VV3 with a relative centrifugal force of about $14 \mathrm{~g}$ ), followed by $60 \mathrm{~s}$ of humidified air, a $30 \mathrm{~s}$ exposure to another odor without shock, and completed by $60 \mathrm{~s}$ exposure to humidified air. If multiple cycles were applied, they followed each other immediately (massed conditioning, Mery et al. 2007b). 3-octanol (OCT) and 4-methylcyclohexanol (MCH) dissolved in paraffin oil were used as odorants. OCT was used at concentration of 0.4 $\mathrm{ml} / \mathrm{l}$; for the first two age classes of the 60 min memory assay the concentration of MHC was $0.6 \mathrm{ml} / 1$; for the remaining assays it was increased to $0.8 \mathrm{ml} / 1$ to correct for the fact that the OCT was more aversive than $\mathrm{MCH}$. 
After a set time elapsed from the end of conditioning ( $5 \mathrm{~min}$ or $60 \mathrm{~min}$ ), the flies were tested for choice between the two odors. An elevator was used to transport them to the central chamber of a T-maze. From that moment they were given $60 \mathrm{~s}$ to choose between the odors, except for 50 day old flies, which were given $75 \mathrm{~s}$ to compensate for their relative sluggishness. The flies choosing each arm of the T-maze (i.e., each odor) were subsequently counted and the proportion of flies choosing octanol versus $\mathrm{MCH}$ was calculated. Flies that remained in the central chamber of the T-maze were excluded from this calculation; thus, differences in locomotor performance did not bias the learning results. The replicate unit in the learning assay consisted of two groups of 50 flies, one conditioned to avoid OCT and the other conditioned to avoid $\mathrm{MCH}$. A value of the memory score was calculated as the difference between the first and the second group in the proportion of flies choosing octanol. The assays were carried out in 6-12 blocks of replicates spread over several days. The memory scores were analyzed using ANOVA with block, diet, number of conditioning cycles, and the interaction between diet and number of cycles as factors (JMP v. 7.0).

\section{Unconditioned response to odors}

The effect of diet on learning performance may be confounded by unconditioned responses to odors. We measured the effect of diet on odor avoidance to test if differences in learning ability were confounded by differences in responses to the odors. Groups of 50 flies were prepared as for the learning assay, but they were not subject to conditioning, but tested in the T-maze for a choice between one of the two odors and the solvent (mineral oil). The odor avoidance score was calculated as $2 p-1$, where $p$ is the proportion of flies choosing the solvent over the odor. Thus, no preference $(p=0.5)$ corresponds to an avoidance score of 0 , and the maximum avoidance score is 1 . For 50day old flies we also carried an assay of unconditioned preference between the two odorants.

\section{Acknowledgments}

We thank D. Fell, U. Friedrich, J. Pont, V. Zrelec for help with the experiments. This work was supported by the Velux Foundation and the Swiss National Science Foundation.

\section{References}

Arking R (2006). The Biology of Aging. Oxford: Oxford University Press.

Austad SN (2004). Is aging programed? Aging Cell. 4, 249-251.

Bellush LL, Wright AM, Walker JP, Kopchick J, Colvin RA (1996). Caloric restriction and spatial learning in old mice. Physiology \& Behavior. 60, 541-547.

Bhandari P, Jones MA, Martin I, Grotewiel MS (2007). Dietary restriction alters demographic but not behavioral aging in Drosophila. Aging Cell. 6, 631-637.

Bond NW, Everitt AV, Walton J (1989). Effects of dietary restriction on radial-arm maze performance and flavor memory in aged rats. Neurobiology of Aging. 10, 27-30.

Brown S, Strausfeld N (2009). The effect of age on a visual learning task in the American cockroach. Learn. Memory. 16, 210-223. 
Burger JMS, Hwangbo DS, Corby-Harris V, Promislow DEL (2007). The functional costs and benefits of dietary restriction in Drosophila. Aging Cell. 6, 63-71.

Burger JMS, Kolss M, Pont J , Kawecki TJ (2008). Learning ability and longevity: a symmetrical evolutionary tradeoff in Drosophila. Evolution. 62, 1294-1304.

Chippindale AK, Leroi AM, Kim SB , Rose MR (1993). Phenotypic plasticity and selection in Drosophila life history evolution.1. Nutrition and the cost of reproduction. J. Evol. Biol. 6, 171-193.

Colomb J, Kaiser L, Chabaud MA, Preat T (2009). Parametric and genetic analysis of Drosophila appetitive long-term memory and sugar motivation. Genes Brain Behav. 8, 407-415.

Davis RL (2005). Olfactory memory formation in Drosophila: From molecular to systems neuroscience. Annu. Rev. Neurosci. 28, 275-302.

Dubey A, Forster MJ, Lal H, Sohal RS (1996). Effect of age and caloric intake on protein oxidation in different brain regions and on behavioral functions of the mouse. Archives of Biochemistry and Biophysics. 333, 189-197.

Edman U, Garcia AM, Busuttil RA, Sorensen D, Lundell M, Kapahi P, Vijg J (2009). Lifespan extension by dietary restriction is not linked to protection against somatic DNA damage in Drosophila melanogaster. Aging Cell. 8, 331-338.

Flood JF , Morley JE (1998). Learning and memory in the SAMP8 mouse. Neuroscience and Biobehavioral Reviews. 22, 1-20.

Forshee BA (2006). The aging brain: is function dependent on growth hormone/insulin-like growth factor-1 signaling? Age. 28, 173-180.

Gould TJ, Bowenkamp KE, Larson G, Zahniser NR, Bickford PC (1995). Effects of dietary restriction on motor learning and cerebellar noradrenergic dysfunction in aged F344 rats. Brain Research. 684, 150-158.

Grandison RC, Piper MDW , Partridge L (2009). Amino-acid imbalance explains extension of lifespan by dietary restriction in Drosophila. Nature. 462, 1061-U1121.

Grotewiel MS, Martin I, Bhandari P, Cook-Wiens E (2005). Functional senescence in Drosophila melanogaster. Ageing Research Reviews. 4, 372-397.

Hashimoto T, Watanabe S (2005). Chronic food restriction enhances memory in mice - analysis with matched drive levels. Neuroreport. 16, 1129-1133.

Hu DY, Cao P, Thiels E, Chu CT, Wu GY, Oury TD, Klann E (2007). Hippocampal long-term potentiation, memory, and longevity in mice that overexpress mitochondrial superoxide dismutase. Neurobiology of Learning and Memory. 87, 372-384.

Ingram DK, Weindruch R, Spangler EL, Freeman JR, Walford RL (1987). Dietary restriction benefits learning and motor performance of aged mice. Journals of Gerontology. 42, 78-81.

Isabel G, Pascual A, Preat T (2004). Exclusive consolidated memory phases in Drosophila. Science. 304, 1024-1027.

Kirkwood TBL, Holliday R (1979). The evolution of aging and longevity. Proc. R. Soc. Lond. B. 205, 531-546.

Lukoyanov NV, Pereira PA, Mesquita RM, Andrade JP (2002). Restricted feeding facilitates time-place learning in adult rats. Behav. Brain Res. 134, 283-290.

Magnusson KR (2001). Influence of diet restriction on NMDA receptor subunits and learning during aging. Neurobiology of Aging. 22, 613-627.

Mair W, Dillin A (2008). Aging and survival: the genetics of life span extension by dietary restriction. Annual Review of Biochemistry. 77, 727-754.

Mair W, Goymer P, Pletcher SD, Partridge L (2003). Demography of dietary restriction and death in Drosophila. Science. 301, 1731-1733.

Mair W, Piper MDW , Partridge L (2005). Calories do not explain extension of life span by dietary restriction in Drosophila. Plos Biology. 3, 1305-1311.

Mallon EB, Brockmann A, Schmid-Hempel P (2003). Immune response inhibits associative learning in insects. Proc. R. Soc. Lond. B. 270, 2471-2473.

Masoro EJ (2005). Overview of caloric restriction and ageing. Mechanisms of Ageing and Development. 126, 913-922.

Means LW, Higgins JL, Fernandez TJ (1993). Midlife onset of dietary restriction extends life and prolongs cognitive functioning. Physiology \& Behavior. 54, 503-508.

Medawar PB (1952). An unsolved problem of biology. London: H. K. Lewis.

Mery F (2007). Aging and its differential effects on consolidated memory forms in Drosophila. Experimental Gerontology. 42, 99-101.

Mery F, Belay AT, So AKC, Sokolowski MB , Kawecki TJ (2007a). Natural polymorphism affecting learning and memory in Drosophila. Proc. Natl. Acad. Sci. USA. 104, 13051-13055.

Mery F, Kawecki TJ (2003). A fitness cost of learning ability in Drosophila melanogaster. Proc. R. Soc. Lond. B. 270 , 2465-2469.

Mery F, Kawecki TJ (2004). An operating cost of learning in Drosophila melanogaster. Anim. Behav. 68, 589-598.

Mery F, Kawecki TJ (2005). A cost of long-term memory in Drosophila. Science. 308, 1148-1148.

Mery F, Pont J, Preat T, Kawecki TJ (2007b). Experimental evolution of olfactory memory in Drosophila melanogaster. Physiol. Biochem. Zool. . 80, 399-405.

Min KJ , Tatar M (2006). Drosophila diet restriction in practice: Do flies consume fewer nutrients? Mechanisms of Ageing and Development. 127, 93-96. 
Miwa S, Riyahi K, Partridge L, Brand MD (2003). Lack of correlation between mitochondrial reactive oxygen species production and life span in Drosophila. In 10th Congress of the International-Association-of-BiomedicalGerontology. (ADN DeGrey, ed^eds). Cambridge, ENGLAND: New York Acad Sciences, pp. 388-391.

Murakami S (2007). Caenorhabditis elegans as a model system to study aging of learning and memory. Mol. Neurobiol. 35, 85-94.

Partridge L, Barton NH (1993). Optimality, mutation and the evolution of ageing. Nature. 362, 305-311.

Partridge L, Barton NH (1996). On measuring the rate of ageing. Proc. R. Soc. Lond. B. 263, 1365-1371.

Partridge L, Pletcher SD, Mair W (2005). Dietary restriction, mortality trajectories, risk and damage. Mechanisms of Ageing and Development. 126, 35-41.

Patel NV , Finch CE (2002). The glucocorticoid paradox of caloric restriction in slowing brain aging. Neurobiology of Aging. 23, 707-717.

Piper MDW, Bartke A (2008). Diet and aging. Cell Metabolism. 8, 99-104.

Piper MDW, Skorupa D , Partridge L (2005). Diet, metabolism and lifespan in Drosophila. Experimental Gerontology. 40, 857-862.

Pitsikas N, Algeri S (1992). Deterioration of spatial and nonspatial reference and working memory in aged rats protective effect of lifelong calorie restriction. Neurobiology of Aging. 13, 369-373.

Pletcher SD (1999). Model fitting and hypothesis testing for age-specific mortality data. J. Evol. Biol. 12, 430-439.

Rose MR (1991). Evolutionary biology of aging. Oxford: Oxford University Press.

Rozing MP, Westendorp RGJ (2008). Parallel lines: nothing has changed? Aging Cell. 7, 924-927.

Sinclair DA (2005). Toward a unified theory of caloric restriction and longevity regulation. Mechanisms of Ageing and Development. 126, 987-1002.

Stewart J, Mitchell J, Kalant N (1989). The effects of life-long food restriction on spatial memory in young and aged Fisher 344 rats measured in the 8-arm radial and the Morris water mazes. Neurobiology of Aging. 10, 669-675.

Tamura T, Chiang AS, Ito N, Liu HP, Horiuchi J, Tully T, Saitoe M (2003). Aging specifically impairs amnesiacdependent memory in Drosophila. Neuron. 40, 1003-1011.

Tatar M, Bartke A, Antebi A (2003). The endocrine regulation of aging by insulin-like signals. Science. 299, 13461351.

Valenzano DR, Terzibasi E, Genade T, Cattaneo A, Domenici L, Cellerino A (2006). Resveratrol prolongs lifespan and retards the onset of age-related markers in a short-lived vertebrate. Current Biology. 16, 296-300.

Vellai T, McCulloch D, Gems D, Kovacs AL (2006). Effects of sex and insulin/insulin-like growth factor-1 signaling on performance in an associative learning paradigm in Caenorhabditis elegans. Genetics. 174, 309-316.

Williams GC (1957). Pleiotropy, natural selection and the evolution of senescence. Evolution. 11, 398-411.

Yamazaki D, Horiuchi J, Nakagami Y, Nagano S, Tamura T, Saitoe M (2007). The Drosophila DCO mutation suppresses age-related memory impairment without affecting lifespan. Nature Neuroscience. 10, 478-484.

Yanai S, Okaichi Y, Okaichi H (2004). Long-term dietary restriction causes negative effects on cognitive functions in rats. Neurobiology of Aging. 25, 325-332.

Table 1. Summary of ANOVA on learning performance results reported in Figures 2 and 3: F-values and significance levels. The analysis was carried out separately for each age class and memory type. All factors were treated as categorical variables, numerator $d f$ follow the number of levels of a given factor -1 .

\begin{tabular}{lcccccc} 
Source & \multicolumn{3}{c}{5 min memory } & \multicolumn{3}{c}{60 min memory } \\
& 5 days & 21 days & 50 days & 5 days & 21 days & 50 days \\
Diet & 0.3 & $6.5^{\star \star \star}$ & $0.2^{\star *}$ & $9.3^{\star \star}$ & 0.6 & 0.0 \\
Number of cycles & $69.9^{\star \star \star}$ & $105.4^{\star \star \star}$ & $5.8^{\star}$ & $44.2^{\star \star \star}$ & $10.5^{\star \star \star}$ & $10.3^{\star \star}$ \\
Diet $\times$ cycles & 1.5 & 0.5 & 0.0 & 2.3 & 0.3 & 0.0 \\
Replicate block & $3.1^{\star *}$ & $1.8 \dagger$ & 0.1 & $2.0^{\star}$ & 0.7 & $2.9 \dagger$ \\
Residual $d f$ & 121 & 121 & 23 & 55 & 55 & 23
\end{tabular}

Significance levels: $\uparrow P<0.1,{ }^{*} P<0.05, * * P<0.01, * * * P<0.001$. 

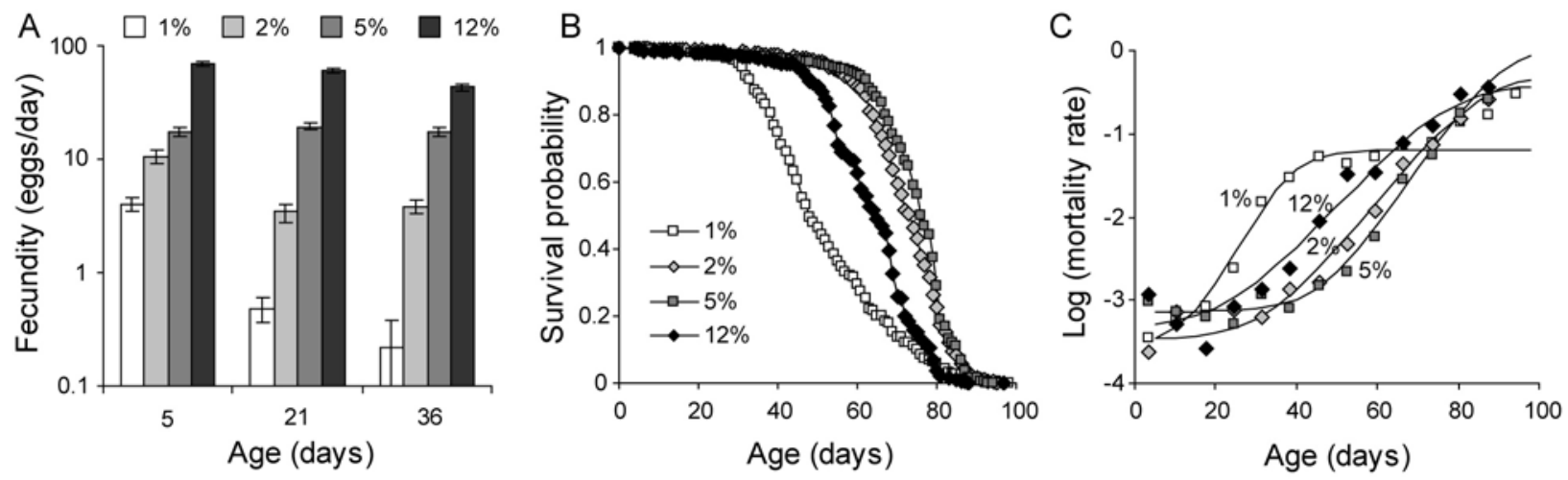

Figure 1. Effect of dietary yeast concentration on fecundity and longevity of once-mated females. (A) Fecundity (mean $\pm \mathrm{SE}$ of the number of eggs laid per day by a female; note the logarithmic scale); the effects of diet $\left(\chi^{2}=9049, d f=3, P<0.0001\right)$, age $\left(\chi^{2}=533, d f=2, P<0.0001\right)$ and their interaction $\left(\chi^{2}=\right.$ 290, $d f=6, P<0.0001$ ) are all significant. $N=27-30$ females per treatment. (B) Product-moment estimates of survival curves (probability of surviving to a given age, given the fly is alive $24 \mathrm{~h}$ after emergence from pupa); $N=6$ cages $\times 200$ females per treatment. (C) Logarithms of daily instantaneous mortality rate (estimated for one-week intervals, for details see Experimental procedures). Lines are logistic-Makeham mortality models fitted with WinModest v. 1.0.2. Proportional hazard analysis (Cox regression) indicates that each survival curve is different from all other curves at the comparison-wise significance level $P<$ 0.001 (smallest pairwise $\chi^{2}=15.0$, d.f. $=1$ ).
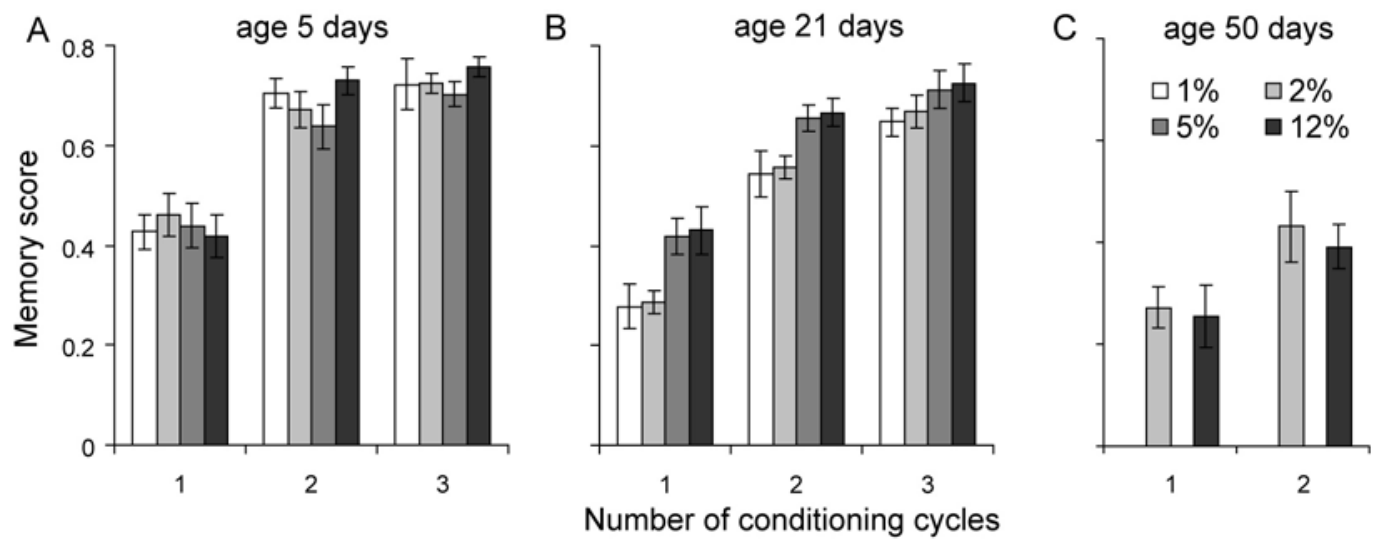

Figure 2. Effect of diet on short-term memory (means \pm SE) of young (A), middle-aged (B) and old (C) flies. $N=10-12$ per diet and cycle number in (A) and (B), $N=6$ in (C). Only $2 \%$ and $12 \%$ diet treatments were represented in the oldest age class (panel C). For statistical analysis see Table 1. 


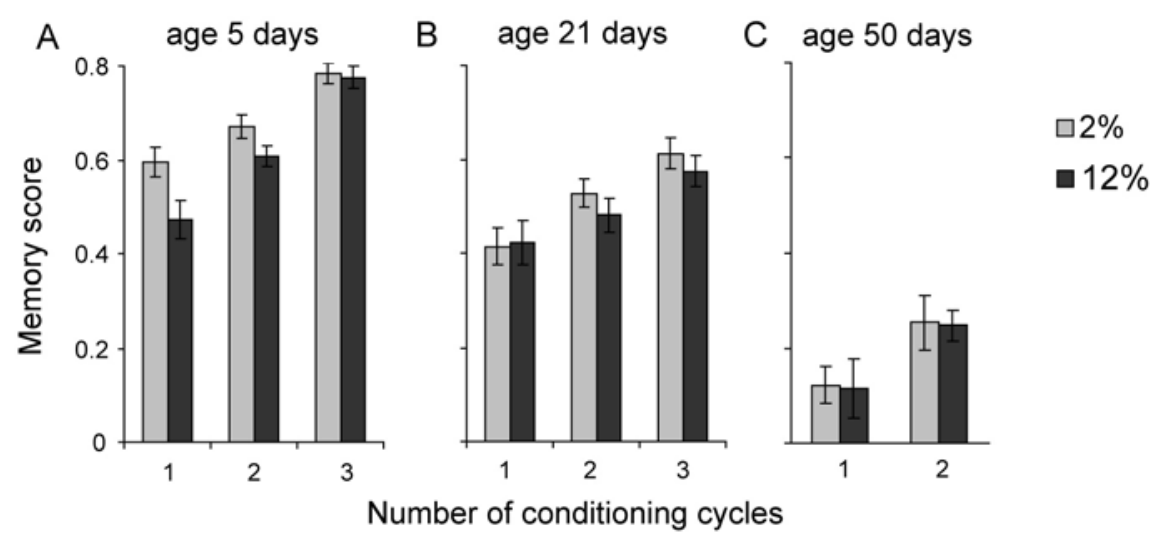

Figure 3 Effect of diet on 60-min memory (means \pm SE) of young (A), middle-aged (B) and old (C) flies. $N=12$ per diet and cycle number in (A) and (B), $N=6$ in (C). For statistical analysis see Table 1.
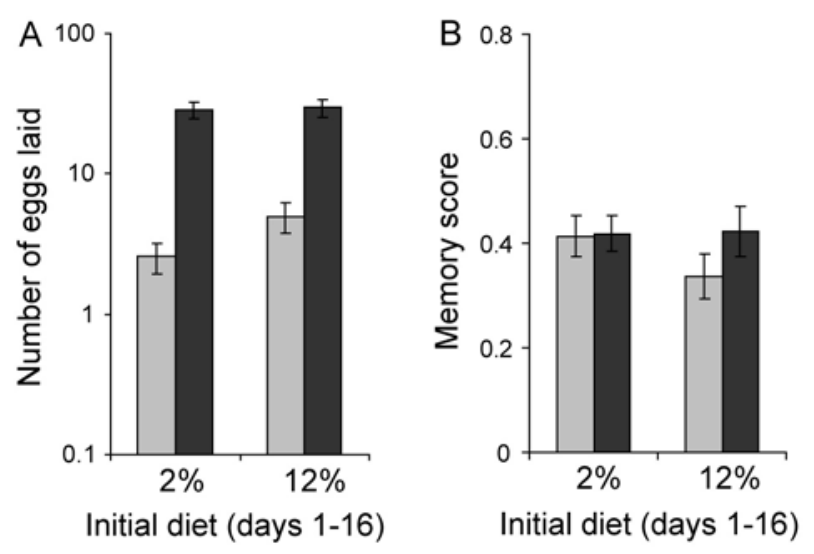

Final diet (days 17-21): $\square 2 \% \quad \square 12 \%$

Figure 4. The effect of diet switch on fecundity (A) and 60 min memory after a single conditioning cycle (B) in 21 day old flies (means \pm SE). "Initial diet" refers to diet offered during the first 17 days of adult life; "final diet" to diet offered for the last four days before the assay. The fecundity data come from an assay independent of that reported in Figure 1, $N=29-30$ per treatment. The learning assay was done in parallel to that reported in Fig. 3; the data for flies continuously maintained on the same diet (initial diet $=$ final diet) are presented in Fig. 3B, but are repeated here for comparison. The memory scores are not significantly different among the treatments $\left(F_{3,33}=1.0, P=0.40\right)$.
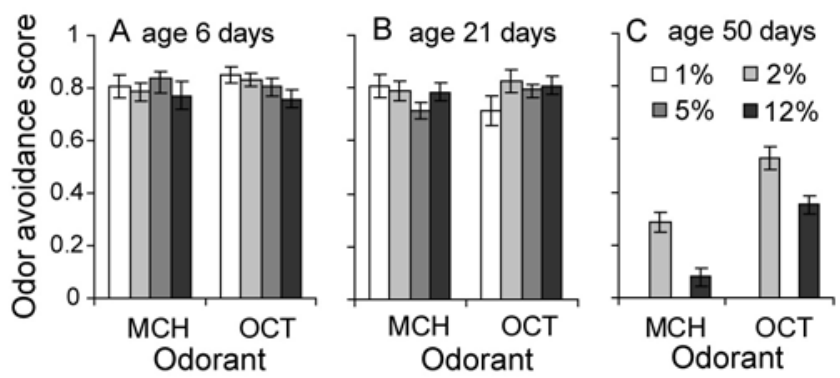

Figure 5. Effect of diet on unconditioned odor avoidance (mean avoidance score $\pm \mathrm{SE}$ ). (A) young flies, $\operatorname{diet} F_{3,77}=1.1, P=0.34, N=12$ per diet and odorant; (B) middle-aged flies, $F_{3,69}=1.1, P=0.35, N=10$ 12; (C) old flies, $\operatorname{diet} F_{1,21}=13.4, P=0.0014, N=8$. 


\section{Dietary restriction affects lifespan but not cognitive aging in Drosophila melanogaster}

Joep M.S. Burger ${ }^{1,2}$, Séverine Büchel ${ }^{1,3}$ and Tadeusz J. Kawecki ${ }^{1}$

\section{Supporting Information}

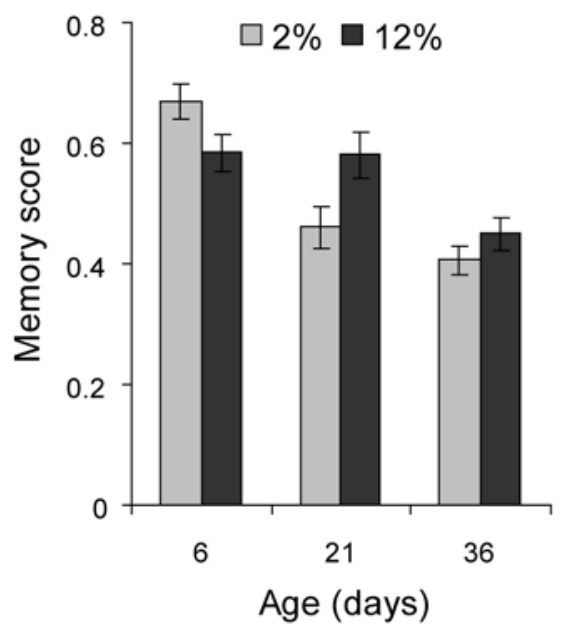

Figure S1. An additional experiment on the effect of diet on 60 min memory after two conditioning cycles at three ages. Age-specific significance of the diet effect: age $6, F_{1,10}=4.9, P=0.052, N=$ 11; age 21, $F_{1,11}=23.7, P<0.001, N=12$; age 36, $F_{1,23}=1.3, P=0.27, N=24$. Diet $\times$ age interaction $F_{2,44}=5.2, P=0.009$. 